\title{
Linear isoforms of the long noncoding RNA CDKN2B-AS1 regulate the c-myc-enhancer binding factor RBMS1
}

\author{
Michael Hubberten ${ }^{1} \cdot$ Gregor Bochenek ${ }^{2} \cdot$ Hong Chen $^{1,3} \cdot$ Robert Häsler $^{4} \cdot$ Ricarda Wiehe $^{1} \cdot$ Philip Rosenstiel $^{4}$. \\ Søren Jepsen ${ }^{2} \cdot$ Henrik Dommisch ${ }^{1} \cdot$ Arne S. Schaefer $\mathbb{B}^{1}$
}

Received: 27 April 2017 / Revised: 5 June 2018 / Accepted: 12 June 2018 / Published online: 14 August 2018

(c) European Society of Human Genetics 2018

\begin{abstract}
Variants in the long noncoding RNA (lncRNA) gene CDKN2B-AS1 (CDKN2B antisense RNA 1; ANRIL) are genomewide associated with type 2 diabetes (T2D), atherosclerosis, and several forms of cancer. However, it is currently not understood how $C D K N 2 B-A S 1$ transcripts translate into diabetes. We previously demonstrated trans-regulation of the proximal polyadenylated transcripts on several genes with functions in glucose and lipid metabolism. However, information on specific genes that are regulated at physiological concentrations by the distal polyadenylated $C D K N 2 B$ $A S 1$ transcripts is lacking. To identify target genes of $C D K N 2 B-A S 1$ trans-regulation, we designed inducible short hairpin RNA constructs and integrated them into the genome of T-Rex HEK293 cells. Changes of gene expression after induction were determined at defined time points by genome-wide mRNA expression analysis. We confirmed downregulation of RBMS1, located on chromosome 2 (RNA-binding motif, single-stranded interacting protein 1) at the transcript and protein level in stable-transfected, inducible HeLa cells, and demonstrated that the effect was independent of the cell type, known $c i s$-regulatory effects, and regulation of the proximal polyadenylated $C D K N 2 B-A S 1$ isoforms. Direct binding of $C D K N 2 B-A S 1$ transcripts to RBMS1 was shown by RNA immunoprecipitation. RBMS1 encodes a cell cycle suppressor. We conclude that the distal and proximal polyadenylated CDKN2B-AS1 transcripts have separate functions in gene regulation, which are independent of the circular CDKN2B-AS1 isoforms and of the genes CDKN2A/2B.
\end{abstract}

Electronic supplementary material The online version of this article (https://doi.org/10.1038/s41431-018-0210-7) contains supplementary material, which is available to authorized users.

Arne S. Schaefer

arne.schaefer@charite.de

1 Department of Periodontology and Synoptic Dentistry, Institute for Dental and Craniofacial Sciences, Charité - University

Medicine Berlin, corporate member of Freie Universität Berlin, Humboldt-Universität zu Berlin, and Berlin Institute of Health, Berlin, Germany

2 Department of Periodontology, Operative and Preventive Dentistry, University of Bonn, Bonn, Germany

3 Department of Stomatology, Zhejiang Provincial People's Hospital, Hangzhou, China

4 Institute of Clinical Molecular Biology, Christian-AlbrechtsUniversity, Kiel, Germany

\section{Introduction}

The long noncoding RNA (lncRNA) CDKN2B-ASI (CDKN2B antisense RNA 1; ANRIL) is genome-wide associated with type 2 diabetes (T2D) [1], several forms of cancer [2, 3], and coronary artery disease (CAD) [4]. $C D K N 2 B-A S 1$ is spliced in a complex pattern but the transcripts can be grouped according to the identity of their last polyadenylated exon, either exon 13 (exons are numbered like NR_047539.1) or 19 (numbered like in NR_003529.3). These transcripts are detectable in a wide variety of tissues but generally non-abundant, with an absolute expression ranging for specific transcripts from 0.01 to one copy per cell [5], indicating a delicate regulation. As an exception to the general low abundance of $C D K N 2 B-A S 1$ transcripts, the $3^{\prime}$ exons (17-19) are expressed at comparably high levels specifically in the intestine (Fig. 1). Additionally, non-polyadenylated circular CDKN2B-AS1 species (circANRIL; exon usage 4-14) are formed in various different cell types [5, 6], which may 


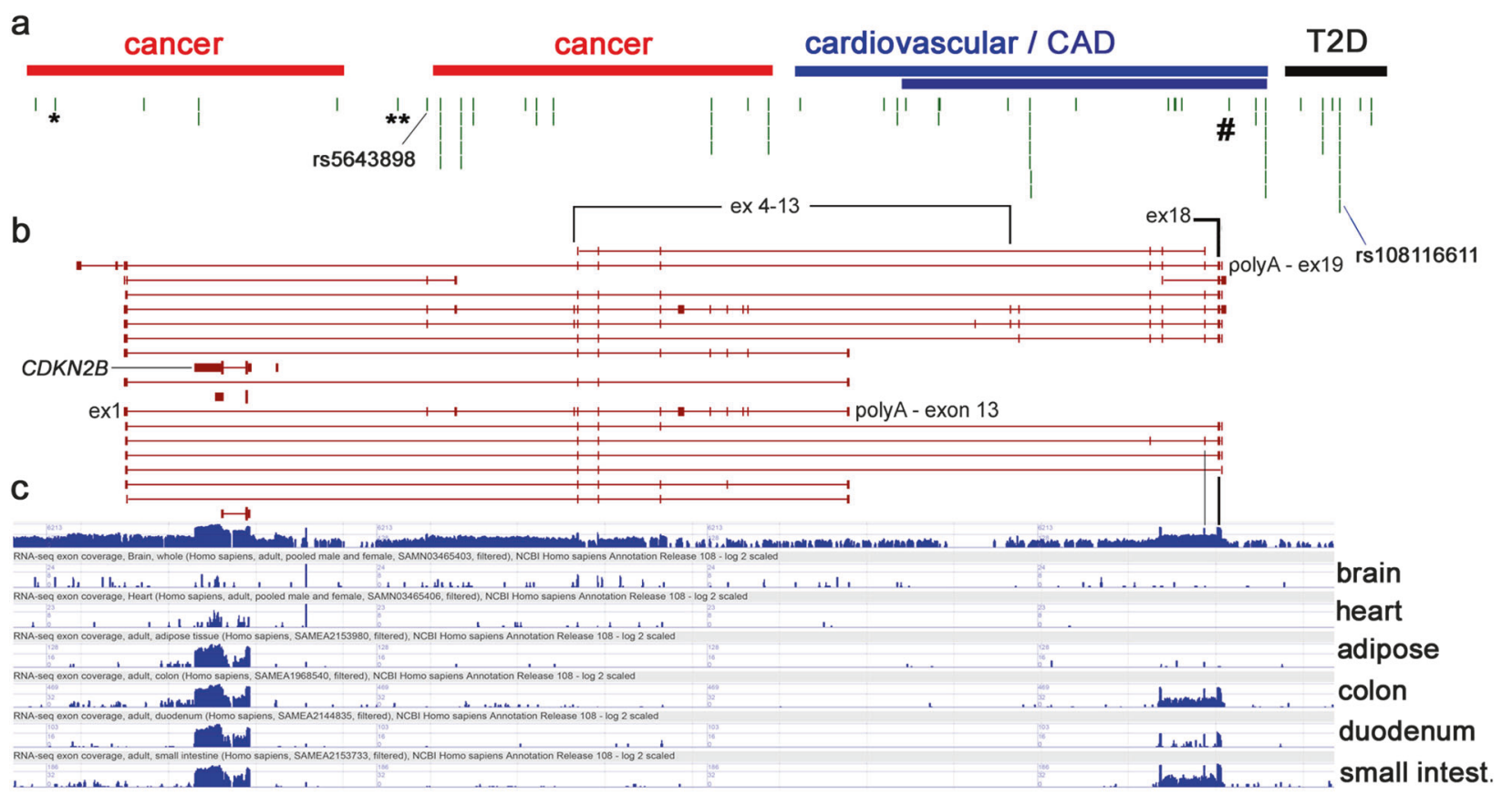

Fig. $1 C D K N 2 B-A S 1$ distal splice forms are strongly expressed in the intestines. $C D K N 2 B-A S 1$ transcripts are spliced in complex and tissuespecific patterns. a GWAS lead SNPs that locate at $C D K N 2 B-A S 1$ as listed in the NHGRI-EBI Catalog of Published GWAS. The upper panel shows the disease phenotypes that relate to the annotated GWAS lead SNPs. The proximal 5' region largely contains cancer-associated variants and spans the short polyadenylated isoforms. The distal region contains risk variants that are associated with cardiovascular diseaserelevant phenotypes. The high-risk CAD haplotype is located at the $3^{\prime}$ end of the long polyadenylated form. The main T2D-associated haplotype, which is independent of the CAD associations, locates well downstream of the coding region of $C D K N 2 B-A S 1$. The CAD highrisk variants and the T2D-associated SNP rs564398, which also shows association with $\mathrm{CAD}$, have regulative effects on the proximal $C D K N 2 B-A S 1$ transcripts but not on CDKN2A (located upstream of

explain the reduced level of the central exons in mature polyadenylated isoforms, being a result of alternative splicing, in which the central exons are skipped. circANRIL was recently demonstrated to regulate rRNA maturation, which leads to activation of p53 and a decrease in cell proliferation [6]. The authors argued that this function of circANRIL may promote atheroprotection, possibly explaining the association of the main-risk CAD haplotype.

Two independent regions $\left(r^{2} \leq 0.05\right)$ at $C D K N 2 B-A S 1$ are genome-wide associated with T2D. One locates proximal in intron 2 and is tagged by SNP rs564398 (GRCh38.p10 chr9:g.22029548T>C), the other is located $3^{\prime}$ outside of the CDKN2B-AS1 exonic region, with tagging SNP rs10811661 (GRCh38.p10 chr9: g.22134095T $>$ C) showing the strongest association in a meta-analyses of T2D GWA studies [1]. Of note, rs564398 is also associated with CAD with similar odds ratio estimates, but it is not in $\mathrm{LD}$ with the CAD
$C D K N 2 B-A S 1$ and not shown on this figure) and $-2 B$. The $3^{\prime}-\mathrm{T} 2 \mathrm{D}$ high-risk variants have no effect on the proximal $C D K N 2 B-A S 1$ transcripts. A specific effect of the $3^{\prime}$-T2D high-risk variants on the distal transcripts is unknown; see also Supplementary Materials, Table 1 for eQTL effects of the GWAS SNPs. * $=$ rs 3731211, associated trait: platelet count; $* *=$ rs615552, migraine; rs564398, T2D, CAD \# = rs10217586, colorectal cancer. b Annotation of known CDKN2B-AS1 transcripts (Ensembl Gene Predictions - archive 75Feb 2014). The positions of exon 1, exons 4-13 (incorporated in circANRIL), and exons 18 (targeted by shRNA) and 19 are indicated (ex =exon). c RNA-seq exon coverage for selected tissues (NCBI Homo sapiens Annotation Release 108, $\log 2$ scaled). High RNA-seq peaks of gastrointestinal tissues correspond with the positions of exons 16-19 (indicated by black lines)

high-risk alleles $\left(r^{2} \leq 0.4\right)$. The distal T2D-associated region, mapping outside of the well-defined CAD-associated haplotype $\left(r^{2} \leq 0.05\right)$ [7], is exclusively associated with T2D, consistent with the hypothesis that both of these T2D and CAD susceptibility effects are independent. rs564398 correlates strongly with the expression of the proximal linear isoforms (exons 1-2), whereas rs10811661 showed no clear correlation with these proximal CDKN2B-AS1 transcripts [8]. To our knowledge, specific correlation with the distal $C D K N 2 B-A S 1$ exons has not been reported for rs 10811661 .

In addition, at the chromosomal position of $C D K N 2 B$ $A S 1$ (chr9p21.3) reside the tumor suppressor genes cyclindependent kinase 2A $(C D K N 2 A)$ and $-2 \mathrm{~B}(C D K N 2 B)$, which have a well-described role to block cell cycle progression [9]. A cis-acting CDKN2B-AS1-mediated silencing mechanism was described to negatively regulate $C D K N 2 A / B$ expression via chromatin remodeling, involving binding of $C D K N 2 B-A S 1$ to the polycomb proteins 
SUZ12 (suppressor of zeste 12 protein homolog) and CBX7 (chromobox 7) [10]. Significant effects on the expression of $C D K N 2 A / B$ were shown for the T2D and CAD-associated SNP rs564398, but the distal T2D and CAD main-risk haplotypes seem to have no effect on $C D K N 2 A / B$ (Supplementary Material, Table 1).

Whereas the functions of $C D K N 2 A / B$ relate to the associations with cancer and circANRIL possibly explains the association of the main-risk CAD haplotype, it is currently not well understood how CDKN2B-AS1 transcripts translate into diabetes. Based on the data described above, we hypothesized in an earlier study that the proximal linear $C D K N 2 B-A S 1$ transcripts have a role in the etiology of diabetes and CAD. We demonstrated a trans-regulatory effect of these isoforms on the genes TMEM258 (transmembrane protein 258; C11ORF10, located at the fatty acid desaturase genes FADS1-3), VAMP3 (vesicle-associated membrane protein 3), and ADIPORl (adiponectin receptor 1), and we confirmed that the observed effects were independent of a specific cell type [11].

In the current study, we aimed to obtain information on specific roles of the distal polyadenylated $C D K N 2 B$ AS1 transcripts and hypothesized independent regulatory functions. To this end, we designed a tetracycline-inducible short hairpin RNA (shRNA) construct to specifically downregulate the distal transcript in physiological concentrations (ex18-shRNA). This construct was integrated into the genome of T-Rex HEK293. We observed the effects of this shRNA construct on genome-wide gene expression after 48 and $96 \mathrm{~h}(\mathrm{~h})$ of induction. Replication of the main findings was performed in T-Rex HeLa cell lines with stable genomic integration of ex18-shRNA. Our data show that moderate reduction of the long CDKN2B-AS1 isoforms significantly reduced RBMS1 (RNA-binding motif, single-stranded interacting protein 1, chromosome 2) on the RNA and the protein level, and that RBMS1 directly binds to CDKN2B-AS1 transcripts.

\section{Material and methods}

\section{Generation of a stable tetracycline-inducible shRNA knockdown system in T-Rex 293 (HEK) and T-Rex HeLa cell lines}

For the polyadenylated long CDKN2B-AS1 transcripts, a total of 19 exons were reported [12]. Whereas the sequence of the polyadenylated exon has variable lengths, the preceding exon is unchanged in all long linear transcript isoforms (Fig. 1). This is why we targeted this exon with our shRNA-construct instead of the adjacent 3 '-polyadenylated exon. Transcript NR_003529.3 (NCBI Reference Sequence) is the longest CDKN2B-AS1 isoform, with 19 exons in total (ENST00000428597.5, $3835 \mathrm{bp}$ ). In NR_003529.3, the exon which we targeted with our shRNA construct is numbered 18. Thus, we labeled this construct ex18-shRNA.

Stable tetracycline-inducible ex18-shRNA T-Rex 293 (HEK) cell lines were constructed using the pENTR $^{\mathrm{TM}}$ / H1/TO vector system (Thermo Fisher Scientific) as described in ref. [13]. Primer sequences are given in the Supplementary Materials. Stable tetracyclineinducible ex18-shRNA T-Rex HeLa cell lines were constructed by transfection of T-Rex HeLa cells (Thermo Fisher Scientific) with the linearized $\mathrm{pENTR}^{\mathrm{TM}}$ / H1/TO-ex18-shRNA vector, using the jetPEI transfection reagent (Polyplus-transfection S.A., USA). Growth conditions for the selection of stable cell lines are given in the Supplementary Materials. Control cell lines (T-Rex HeLa) targeting ex13-shRNA were constructed as described in ref. [13].

\section{Expression analyses}

Five biological replicates of T-Rex 293 (HEK) ex18shRNA cells were harvested 0,48 , and $96 \mathrm{~h}$ after induction with tetracycline. Affymetrix Human Gene 1.0 ST Arrays were hybridized and analyzed as previously described [13], following the MIAME standards. Data were submitted to Gene Expression Omnibus (GEO, http://www. ncbi.nlm.nih.gov/geo, series: GSE111844; samples: GSM3040967-GSM3040981). shRNA expression of T-Rex Hela ex18-shRNA and T-Rex Hela ex13-shRNA cells was induced by adding $1 \mu \mathrm{g} / \mathrm{ml}$ tetracycline to the growth medium at a confluency of $30 \%$. For the qRTPCR, five biological replicates of treated and untreated cells were harvested 24 and $48 \mathrm{~h}$ after tetracycline induction. The qRT-PCR protocol is described in the Supplementary Material.

\section{Western blot}

T-REX 293 HEK and HeLa cells were grown in three independent cultures. shCDKN2B-AS1 constructs were induced for $96 \mathrm{~h}$ with tetracycline in triplicates and the protein blots were repeated for each culture three times. The western blot protocol is described in the Supplementary Material.

\section{RNA immunoprecipitation (RIP)}

RIP was performed according to the protocol described in ref. [14] with some modifications specified in the Supplementary Material. 
Table 1 Reduction of transcript levels of $C D K N 2 B-A S 1$ and the selected candidates in the explorative step

\begin{tabular}{lll}
\hline Transcript & $\begin{array}{l}\text { Fold reduction }(48 \mathrm{~h} \\
\text { ex18-shRNA) }\end{array}$ & $\begin{array}{l}\text { Fold reduction (after 96 } \\
\mathrm{h} \text { ex18-shRNA) }\end{array}$ \\
\hline $\begin{array}{l}\text { CDKN2B-AS1 } \\
\text { (exon 18) }\end{array}$ & 1.87 & 1.75 \\
RBMS1 & 1.31 & 1.40 \\
OCR1 & 1.58 & 1.37 \\
\hline
\end{tabular}

\section{Results}

\section{Induced knockdown of exon 19 CDKN2B-AS1 transcripts is correlated with decreased levels of \\ RBMS1 and OCR1 in HEK cells but not with CDKN2A and $-2 B$}

To quantify the reduction of shRNA-mediated knockdown of CDKN2B-AS1 exon 18 upon tetracycline induction, we assessed the transcript levels in our inducible knockdown HEK and HeLa cell systems at two different time points after tetracycline induction. In HEK cells, exon 18 transcript levels decreased to a minimum after $48 \mathrm{~h}$ tetracycline induction of the ex18-shRNA, with a 1.87 -fold reduction compared with the unstimulated controls. The reduction of transcripts remained stable with a 1.75 -fold decrease after $96 \mathrm{~h}$ of tetracycline-induced shRNA silencing (Table 1). These effects on transcript levels were comparable to our previous study, which employed a shRNA construct to specifically silence exon 13, termed ex13-shRNA [13]. Having proved the functionality of our construct, we performed genome-wide mRNA profiling in the ex18-shRNA-HEK cell lines using Affymetrix HG 1.0 ST Arrays as previously published. Five replicates were performed at each of the time points 0 , 48 , and $96 \mathrm{~h}$ (h) following tetracycline induction. Protein coding genes were selected for follow-up studies using the following pre-assigned selection criteria: False discovery rate (FDR) $<5 \%$, fold change of expression $>1.3$ at both time points; no outliers accepted. Two transcripts, OCRI (ovarian cancer-related protein 1) and RBMS1 (RNA-binding motif, single-stranded interacting protein 1) showed significant reduction of transcript levels after 48 and $96 \mathrm{~h}$ of induction.) (Table 1 and Supplementary Material, Table 2). None of the upregulated genes passed the selection criteria.

In addition to RBMSI and $O C R 1,27$ genes were $>1.3-$ fold downregulated after $48 \mathrm{~h}$ of tetracycline induction of the ex18-shRNA (FDR $<5 \%$ ) and 9 genes were $>1.3$-fold downregulated after $96 \mathrm{~h}(\mathrm{FDR}<5 \%)$. Upregulation $(>1.3-$ fold) was observed for 16 genes after $48 \mathrm{~h}(\mathrm{FDR}<5 \%)$ and for no gene after $96 \mathrm{~h}(\mathrm{FDR}<5 \%)$ (Supplementary Material, Table 3). To confirm the effects of ex18-shRNA on the expression of $O C R 1$ and $R B M S 1$, we performed an independent replication of the experiment $(N=5)$. Because the strongest effects were observed in the explorative stage after $48 \mathrm{~h}$, we quantified the transcript levels at that time, using qRT-PCR. After $48 \mathrm{~h}$ tetracycline induction of the ex18-shRNA, OCRI and RBMS1 showed significant reduction of transcript levels (OCRI: 2.6-fold, $P=0.0075$; RBMS1: 1.7-fold, $P=0.022$; $C D K N 2 B-A S 1$ exon 19: 2.4fold, $P=0.0042$; Fig. 2).

$C D K N 2 B-A S 1$ transcripts were not represented on the HG 1.0 ST Arrays. Therefore, we used qRT-PCR to test if the reduced transcript levels of the long linear isoforms have an effect on splice forms, which use polyadenylated exon 13. After $48 \mathrm{~h}$ of ex18-shRNA induction, $C D K N 2 B-A S 1$ splice variant containing exon 13 were downregulated 2.1-fold. This was different to our earlier observations, which showed that ex13-shRNA expression had no effect on the long polyadenylated CDKN2B-ASI transcripts [13].

A negative correlation of the concentration of the proximal transcripts of $C D K N 2 B-A S 1$ and $C D K N 2 A$ and $-2 B$ expression was earlier described [10] and likewise, our previous exon 13-shRNA-construct that specifically downregulated transcripts that terminated with polyadenylated exon 13, showed low but significant increase in transcript levels of CDKN2A (1.5-fold) and -2B (1.3-fold) after $96 \mathrm{~h}$ of induction. In contrast, the ex18-shRNA constructs did not change $C D K N 2 A$ and $C D K N 2 B$ transcript levels after 48 and $96 \mathrm{~h}$ of induction in the stable integrated HEK cells.

\section{Induced knockdown of exon 19 CDKN2B-AS1 transcripts correlates with decreased RBMS1 expression in HeLa cells}

To validate our findings in an independent cell type and to exclude false-positive findings due to cell-linespecific artifacts, we established a HeLa cell line with the ex18-shRNA construct stable integrated into the genome. In the current as well as in the earlier study, which used the ex13-shRNA in HEK cells, we observed the strongest effects after $48 \mathrm{~h}$ of shRNA expression. This is why we quantified the transcript levels at that time point. To observe potential earlier effects, we additionally monitored gene expression after $24 \mathrm{~h}$ of ex 18 -shRNA induction. Five replicates were performed and expression was quantified by qRT-PCR.

ex18-shRNA expression resulted in a 1.5- and 1.3-fold downregulation of the long polyadenylated $C D K N 2 B-A S 1$ transcripts after $24 \mathrm{~h}$ and $48 \mathrm{~h}$, respectively (Fig. 3). Similar to our results in HEK cells, RBMS1 transcript levels were significantly reduced 1.3 -fold and 1.4 -fold after $24 \mathrm{~h}$ and $48 \mathrm{~h}$, respectively, of ex18-shRNA expression. 

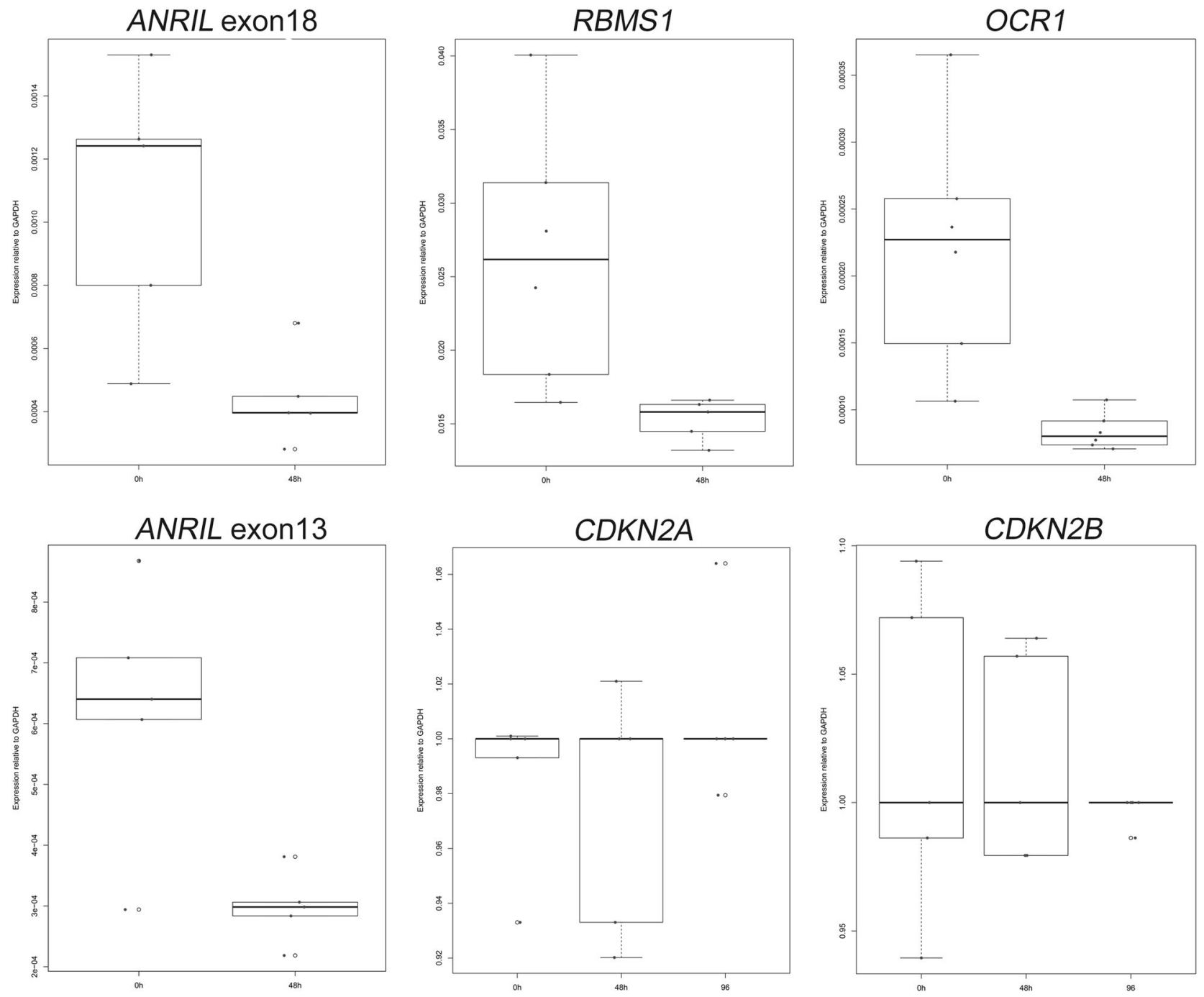

Fig. 2 mRNA expression levels of RBMS1, OCR1, and $C D K N 2 A / 2 B$ in HEK cells before and after ex18-shRNA induction. Transcript levels of $C D K N 2 B-A S 1$ exon 18 are shown as a control for the functionality of the stable integrated ex18-shRNA construct. Significant reduction $(P<0.05)$ of RBMS1 and OCR1 but also for $C D K N 2 B-A S 1$ exon 13

was observed $48 \mathrm{~h}$ after ex18-shRNA induction. CDKN2A/2B expression is not changed after $48 \mathrm{~h}$ or at a later time point. The data were generated as a biological replication of the results of the explorative step (HG 1.0 ST arrays) using qRT-PCR

Likewise, $C D K N 2 A$ and $-2 B$ were expressed at comparable levels before and after ex18-shRNA induction. No difference in OCRl expression was detected in HeLa cells at either time point. The short polyadenylated $C D K N 2 B-A S 1$ transcripts showed a reduction of 1.5 -fold at time point $24 \mathrm{~h}$ but showed no difference of transcript levels after $48 \mathrm{~h}$.

To rule out that our observations were produced by unspecific effects of the shRNA expression or because of the tetracycline treatment, we analyzed RBMSI mRNA concentrations by qRT-PCR in T-Rex HeLa cells with a stable integrated ex13-shRNA. No difference in RBMS1 expression was observed after tetracycline induction. Likewise, no reduction of RBMS1 transcript levels was observed after knockdown of the short $C D K N 2 B$-AS1 splice variant in T-Rex HEK cells carrying the ex13-shRNA [13].

\section{Knockdown of the long CDKN2B-AS1 splice variant reduces RBMS1 protein content}

To validate the effects of reduced concentrations of $C D K N 2 B-A S 1$ exon 19 isoforms on the protein level of $R B M S 1$, we performed western blot analysis with our TREX 293 HEK and HeLa cells inducible overexpressing ex18-shRNA. Following $96 \mathrm{~h}$ of ex18-shRNA induction, RBMS1 protein concentration invariably showed distinct reduction in HEK as well as in HeLa cells in each of the three independent cell cultures, which were grown for both cell lines (Fig. 4). RBMS1 contains two RNA-binding domains [15]. Therefore, we tested if RBMS1 physically interacts with $C D K N 2 B-A S 1$ transcripts. We performed RNA immunoprecipitation (RIP) using RBMS1 antibodies 
Linear isoforms of the long noncoding RNA CDKN2B-AS1 regulate the c-myc-enhancer binding factor RBMS1

85

as baits. When we used RBMS1 as a bait, $C D K N 2 B-A S 1$ exon 13 and exon 18 transcripts were enriched 76-fold and 63-fold, respectively (Fig. 4). GAPDH enrichment was used as background control and displayed a fivefold enrichment in RBMS1-RIP in comparison to no-antibody control RIP. However, CDKN2B-AS1 enrichment was significantly
ANRIL exon 18

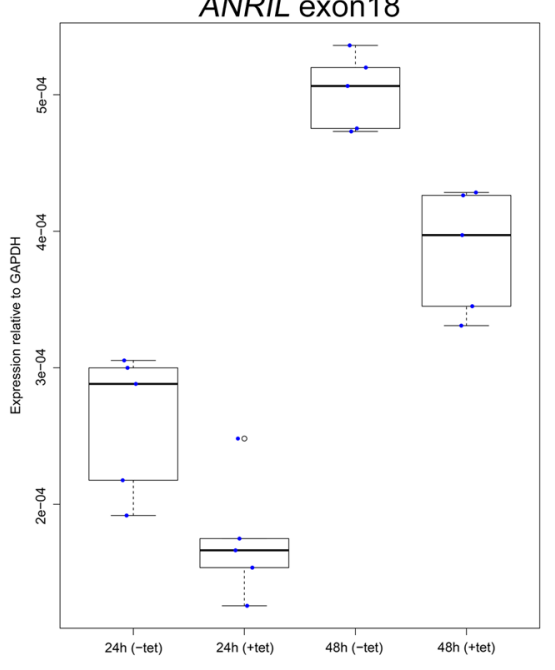

ANRIL exon 13

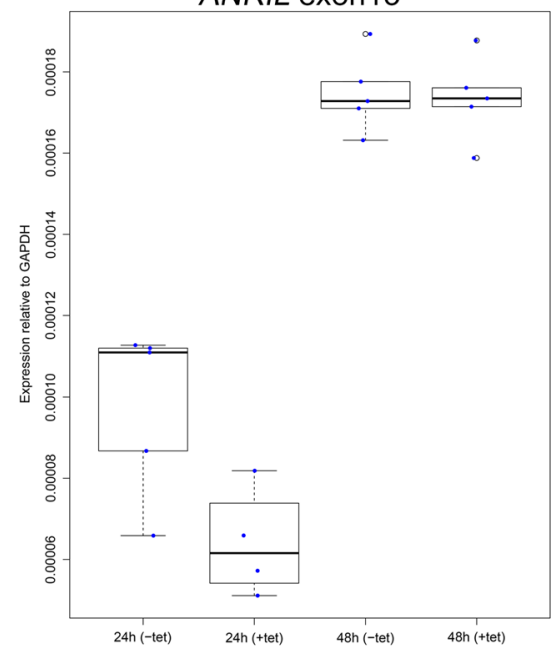

REMS

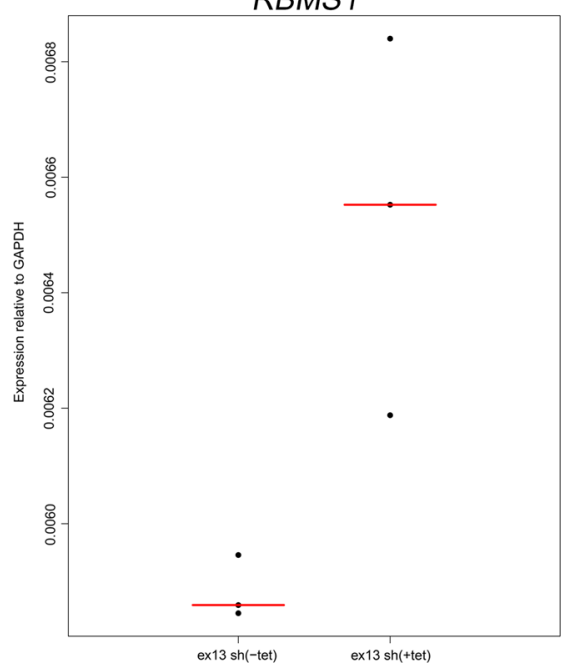

REMS

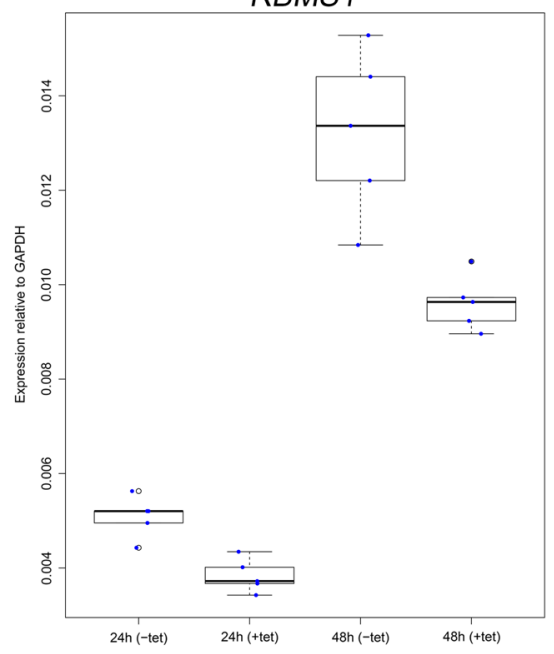

CDKN2A

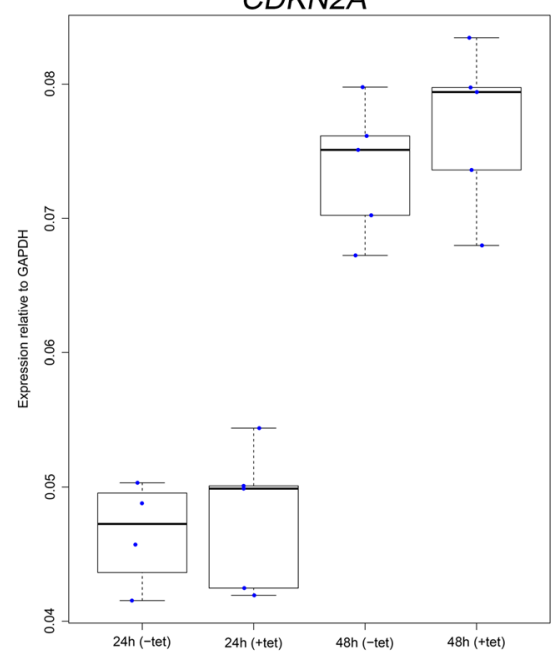

ANRIL exon 18

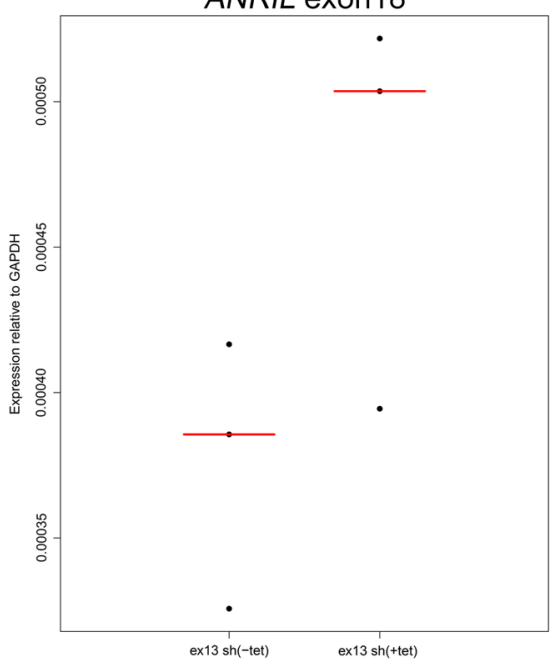

OCR 1

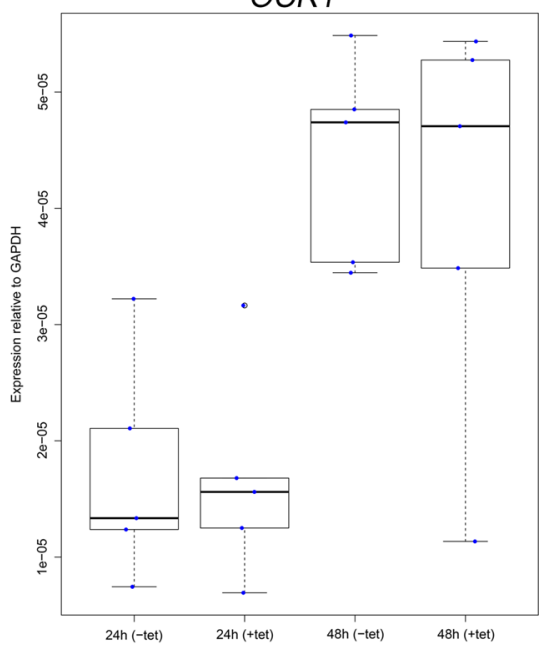

CDKN2B

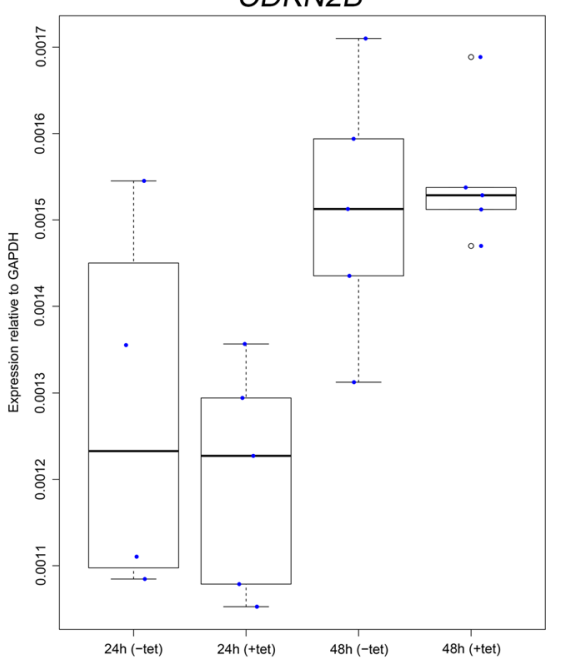

ANRIL exon 13

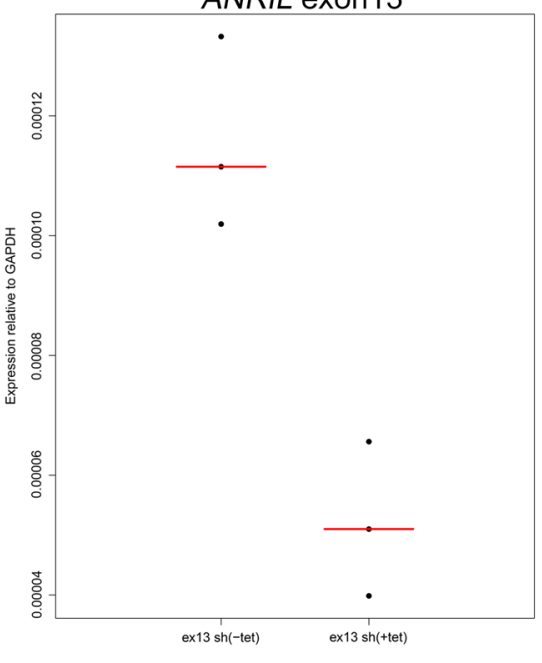

SPRINGER NATURE 
Fig. 3 mRNA expression levels of RBMS1, OCR1, and $C D K N 2 A / 2 B$ in HeLa cells. a-f Before and after ex18-shRNA induction. a Transcript levels of $C D K N 2 B-A S 1$ exon 18 are shown as a control for the functionality of the stable integrated ex18-shRNA construct. b, c Significant reduction $(P<0.05)$ of $R B M S 1$ but not for $O C R 1$ was observed 24 and $48 \mathrm{~h}$ after ex18-shRNA induction. d CDKN2B-AS1 exon 13 levels were significantly reduced after $24 \mathrm{~h}$. e, f CDKN2A and $-2 \mathrm{~B}$ expression was not changed at any time point. $\mathbf{g}-\mathbf{i}$ Before and after ex13-shRNA induction. $\mathbf{g}, \mathbf{h}$ RBMS1 and CDKN2B-AS1 exon 18 levels were not reduced after ex13-shRNA induction. (see also Supplementary Materials, Fig. 1, which confirms the lack of an effect of exon 13 knockdown on RBMS1 in Heck cells, which have the ex13shRNA construct stable integrated), i Transcript levels of $C D K N 2 B$ $A S 1$ exon 13 are shown as a control for the functionality of the stable integrated ex13-shRNA construct

higher than GAPDH enrichment. We used CBX7 and SUZ12 antibodies as positive controls, because immunoprecipitation of $C D K N 2 B-A S 1$ was reported for both proteins $[10,16,17]$. Although both antibodies significantly enriched the $C D K N 2 B$ - $A S 1$ transcripts in the RIP experiments in comparison to the no-antibody control, only $\mathrm{CBX} 7$ displayed significant enrichment of $C D K N 2 B-A S 1$ splice variant 13 and 19, when compared to the background control GAPDH. Only marginal enrichment of $C D K N 2 B$ AS1 transcripts and GAPDH could be observed in an immunoglobulin control with ACTIN antibody. Finally, we confirmed the pulldown of RBMS1 in a protein blot of the RBMS1-RIP-precipitated proteins.

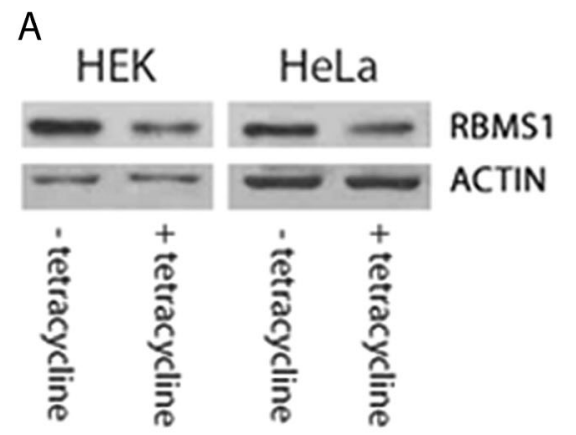

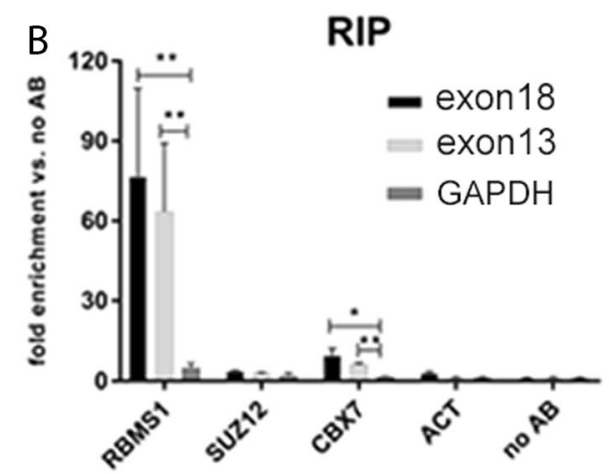

C

RBMS1-RIP

ACTIN-RIP

In the current study, we analyzed the effects of the distal $C D K N 2 B-A S 1$ transcripts on gene regulation to improve the understanding of how the different polyadenylated linear transcripts may be relevant to the various disease associations at this locus. We give proof that the expression of these transcripts is positively correlated with $R B M S 1$ on the RNA and protein levels and that $C D K N 2 B-A S 1$ transcripts bind to the RBMS1 protein.

RBMS1 was first identified as a protein that binds to an enhancer element of the protooncogene $M Y C$ (v-myc avian myelocytomatosis viral oncogene) [15]. MYC encodes a transcription factor, which is delicately regulated due to its central role in cell proliferation and apoptosis. Accordingly, mutations and overexpression of $M Y C$ have been associated with a variety of tumors as recently reviewed [18]. A repressor of c-myc transcription is P53 [19]. We note that overexpression of circANRIL transcripts was recently demonstrated to induce p53 transcription. Interestingly, CDKN2B-AS1 is associated at genome-wide significance with several forms of cancer. Adjacent to the association of rs10217586 (hg19 chr9:g.22121099A>T) with colorectal cancer locate the GWAS lead SNPs of T2D. We note that genome-wide significant associations with T2D were also described for the chromosomal region of RBMS1 [20]. The T2D GWAS lead SNP rs7593730-T (hg38.p10, chr2:

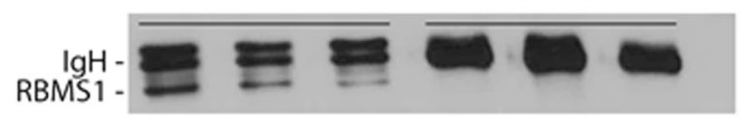

Fig. 4 The long polyadenylated CDKN2B-AS1 transcripts interact with RBMS1. a RBMS1 protein concentration is reduced after $96 \mathrm{~h}$ knockdown of CDKN2B-AS1 exon 18 in HEK and HeLa cells. b RBMS1 RNA immunoprecipitation in HeLa cell extract. Antibodies for RBMS1, SUZ12, CBX7, and ACTIN and no-antibody control were used for RNA immunoprecipitation (RIP). RNA precipitation of $C D K N 2 B$-AS1 exons 13 and -18 isoforms and of GAPDH was measured by qRT-PCR. Data are shown as enrichment in comparison to no-antibody RIP. Both $C D K N 2 B-A S 1$ splice variants were strongly enriched in the RBMS1-RIP (ex13 =63-fold, ex18=76-fold) and to a lesser extent in the CBX7-RIP (ex13=6-fold, ex18=10-fold) in comparison to the no-antibody RIP. The enrichment was significantly higher than enrichment of the GAPDH background control (GAPDH in CBX7-RIP 1.5-fold, in RBMS1-RIP 4.8-fold). Enrichment of the $C D K N 2 B-A S 1$ exons 13 and 18 isoforms were also observed in the SUZ12 RIP (ex13 $=3.2$-fold, ex18 $=3.7$-fold) relative to the noantibody control, but it was not significantly higher compared to enrichment of GAPDH. ACTIN RIP was included as an immune globuline control. c Protein extracts of the RBMS1-RIP and ACTINRIP were analyzed by western blotting, showing the presence of RBMS protein in the RBMS1-RIP extract but not in the ACTIN-RIP extract 
12:g.160314943T $>$ C ; intronic to $R B M S 1)$ as well as other variants with strong $\operatorname{LD}\left(r^{2} \geq 0.8\right)$, showed cis-effects on the expression of RBMS1 in blood [21], peripheral blood monocytes [22], mononuclear cells [23], and the cerebellum of Alzheimer's disease patients [24]. Although not significant on a study-wide level, these data suggest that the T2D-associated haplotype block may have a cis-regulatory effect on the expression of $R B M S 1$, indicating a link of RBMS1 function and diabetes. However, while this protein is implicated in DNA replication, gene transcription, cell cycle progression, apoptosis, and the regulation of mRNA degradation and translation [25], to our knowledge, a role of RBMS1 in diabetogenic pathways had been reported only by the GWAS study that identified the association of rs7593730 with T2D. This study additionally reported a nominal significant effect of the rs7593730-T allele on fasting glucose concentrations, but emphasized the exploratory nature of this observation. This also holds for the reports of the eQTL studies, and we emphasize the need for a more extensive experimental and clinical follow-up before a mechanism that links RBMS1 action with T2D can be proposed.

In the initial screening experiment performed in HEK cells, the transcript levels were monitored by genome-wide expression profiling after 48 and $96 \mathrm{~h}$ following induction of the shRNA knockdown construct. We targeted exon 18 instead of exon 19 with the shRNA because the sequence of exon 18 is unchanged in all long linear transcript isoforms, whereas exon 19 is variable in length (Fig. 1). By selecting exon 18 as a target for shRNA silencing, we believe to have captured the information of this group of linear transcripts comprehensively. Apart from RBMS1, only one additional gene, OCR 1 , showed consistent changes of expression of $>1.3$-fold at both time points, which were subsequently validated by qRT-PCR. The replication in HeLa cells failed to give evidence of an effect of CDKN2B-ASl transcript levels on OCR1. This gene, which currently has an unknown function, was first identified in screening for differential expressed genes in ovarian cancer [26]. In the light of the colorectal cancer association with SNP rs10217586 (GRCh38.p10 chr9:12:g.22121350A >T), it could be possible that a molecular context of $C D K N 2 B-A S 1$ and $O C R 1$ might exist. In this case, the non-validation in HeLa cells could be due to potential cell type and tissuespecific differences between HeLa and HEK cells although our study does not provide evidence for this hypothesis.

In addition to RBMSI and $O C R I$, no other gene fulfilled our pre-assigned selection criteria to be carried forward to validation in a different cell type. This could be a consequence of the comparatively moderate reduction of transcript levels, which was within a physiological range but may have been too weak to detect additional effects on gene expression in our in vitro cell models. Thus, the prevention of artificially high transcript levels or a complete knockout, which we considered to avoid artifacts and type I errors caused by unnatural compensatory reactions, may also have been a limitation of our study, because it could increase the chances of false negatives.

Following ex18-shRNA induction, we observed that exon 19 and exon 13 polyadenylated $C D K N 2 B$ - $A S 1$ transcripts were downregulated. To assess if RBMS1 reduction was specific to the knockdown of the long splice variants, we analyzed RBMS1 transcript levels in HeLa T-Rex cells, with the ex13-shRNA construct stable integrated into the genome. After induction of the ex13-shRNA, no changes in RBMS1 expression were observed. Likewise, no change of RBMS1 transcript levels was detected when reassessing our earlier generated expression data of HEK293 cells that inducible expressed the stable integrated ex13-shRNA construct [11]. Accordingly, we conclude that the decrease of RBMS1 transcripts is a specific response to the ex18-shRNA-mediated knockdown of the long $C D K N 2 B$ AS1 splice variant.

Because CDKN2B-AS1 isoforms that terminate with polyadenylated exon 13 do not carry exon 18, the mechanisms underlying the correlated reduction of both polyadenylated splice variants after ex18-shRNA induction is speculative. An explanation could be a compensatory mechanism that adjusts the ratio of polyadenylated CDKN2B-AS1 19 transcripts. It is presumed that each type of cell actively controls the relative quantities of alternative splice isoforms to maintain cellular homeostasis. Given the important role of $C D K N 2 B$-AS1 transcripts in controlling cell growth, its expression should be precisely regulated. Likewise, different CDKN2B-AS1 isoforms were earlier reported to be positively correlated with each other [17], and the intestines maintain strong expression of exons 16-19 in parallel to underrepresentation of proximal introns (see Fig. 1). It is possible that a reduction of the long polyadenylated isoforms is recognized by the cell, leading to increased splicing of the long $C D K N 2 B-A S 1$ isoforms. In consequence, this would result in a reduction of the short transcripts. In agreement with this, splice isoform ratios were shown to be exceptionally robust to genetic and environmental variability in the mouse model [27]. If cellular homeostasis required a higher ratio of the long polyadenylated isoforms to the short, this would also explain why we did not observe a reciprocal compensation, when we reduced the short polyadenylated transcripts.

Proximal CDKN2B-AS1 transcripts (exons 1-2) and circANRIL (exons 5-7) were reported to cis-regulate $C D K N 2 A / B$ expression $[10,16]$. Accordingly, it can be speculated that RBMS1 downregulation is a consequence of changes in $C D K N 2 A / B$ expression in response to reduced $C D K N 2 B-A S 1$ transcript levels. To investigate this possibility, we evaluated the transcriptional response to 
$C D K N 2 A / B$ in HEK and HeLa cells after knockdown of the long $C D K N 2 B$-AS1 splice variants. After ex18-shRNA expression, we observed no effect on $C D K N 2 A / B$ transcript levels. These data demonstrate that the downregulation of $R B M S 1$ is independent of $C D K N 2 A / B$ expression.

To show that RBMS1 physically interacts with $C D K N 2 B$ AS1 transcripts, we performed a RIP experiment and were able to pull down RBMS1 complexes with an anti-RBMS1 antibody. In this complex, the long as well as the short polyadenylated $C D K N 2 B$ - $A S 1$ splice variants were detected using primers specific for exons 18 and 13, respectively. This gave proof for a direct binding of RBMS1 to $C D K N 2 B$ $A S 1$. It also indicated that both isoforms were present in the RBMS1 complex and consequently, both isoforms may directly bind to RBMS1. Additionally, $C D K N 2 B-A S 1$ was more efficiently pulled down in the RIP using the RBMS1 antibody compared to SUZ12 or CBX7 antibodies, indicating a higher occupancy or more stable binding of $C D K N 2 B$ AS1 to RBMS1. Because the transcript levels of RBMS1 only correlated with the long polyadenylated isoform, we suggest that the correlation of $C D K N 2 B-A S 1$ and $R B M S 1$ transcripts is not mediated by the binding of $C D K N 2 B-A S 1$ mRNA to RBMS1 protein but indicates an independent regulatory mechanism.

A critical requirement for the binding of RBMS1 to $C D K N 2 B-A S 1$ is that they co-localize in the same cellular compartment. CDKN2B-AS1 transcripts were detected in the nucleus by several studies [6, 10, 16, 28], and binding of RBMS1 to nuclear double-stranded DNA was experimentally shown for a DNA replication origin/transcriptional enhancer of the human c-myc gene [25]. This gives evidence for the RBMS1 protein to be localized in the nucleus, where it would be able to interact with $C D K N 2 B$ AS1 transcripts.

We note that an earlier study, which had constitutively overexpressed $C D K N 2 B$-AS1-ex 19 transcripts in HEK cells, did not report different transcriptional regulation of RBMS1. However, a constitutive overexpression is likely to induce a variety of compensatory effects, which might mask the primary response of the overexpression of $C D K N 2 B-A S 1$. Here, an induced knockdown at physiological levels may be advantageous compared to a constitutive overexpression, which was the reason for performing the current study.

In summary, we provide evidence for a mechanistic link of $C D K N 2 B-A S 1$ function and RBMS1 activity. Together with our earlier findings, we conclude that the proximal and distal polyadenylated $C D K N 2 B-A S 1$ transcripts have independent functions in the regulation of distant genes, which comprise TMEM258, VAMP3, ADIPOR1, and RBMS1, respectively. These functions are independent of the circANRIL isoforms, which have a role in atherosclerosis and are independent of the genes $C D K N 2 A / 2 B$, which have a role in cancer. Future studies will further validate the specific function of CDKN2B-AS1 in the gastrointestinal tract, where the distal exons are predominantly expressed, and in insulin-responsive and -releasing tissues.

Acknowledgements We gratefully acknowledge the contributions of the technicians Dorina Ölsner (IKMB, Christian-Albrechts-Universität zu Kiel), Diana Lalaouni (Department of Periodontology, Operative and Preventive Dentistry, Rheinische-Friedrich-Wilhelms-Universität Bonn), and Verena Kanitz (Department of Periodontology and Synoptic Dentistry, Charité - University Medicine Berlin).

Funding This work was supported by a research grant of the German Research Foundation DFG (SCHA 1582/1-2).

\section{Compliance with ethical standards}

Conflict of interest The authors declare that they have no conflict of interest.

\section{References}

1. Zeggini E, Weedon MN, Lindgren CM, et al. Replication of genome-wide association signals in UK samples reveals risk loci for type 2 diabetes. Science. 2007;316:1336-41.

2. Cheng TH, Thompson D, Painter J, et al. Meta-analysis of genome-wide association studies identifies common susceptibility polymorphisms for colorectal and endometrial cancer near SH2B3 and TSHZ1. Sci Rep. 2015;5:17369.

3. Shete S, Hosking FJ, Robertson LB, et al. Genome-wide association study identifies five susceptibility loci for glioma. Nat Genet. 2009;41:899-904.

4. Wellcome Trust Case Control C. Genome-wide association study of 14,000 cases of seven common diseases and 3,000 shared controls. Nature. 2007;447:661-78.

5. Burd CE, Jeck WR, Liu Y, Sanoff HK, Wang Z, Sharpless NE. Expression of linear and novel circular forms of an INK4/ARFassociated non-coding RNA correlates with atherosclerosis risk. PLoS Genet. 2010;6:e1001233.

6. Holdt LM, Stahringer A, Sass K, et al. Circular non-coding RNA ANRIL modulates ribosomal RNA maturation and atherosclerosis in humans. Nat Commun. 2016;7:12429.

7. Broadbent HM, Peden JF, Lorkowski S, et al. Susceptibility to coronary artery disease and diabetes is encoded by distinct, tightly linked SNPs in the ANRIL locus on chromosome 9p. Hum Mol Genet. 2008;17:806-14.

8. Cunnington MS, Santibanez Koref M, Mayosi BM, Burn J, Keavney B. Chromosome 9p21 SNPs associated with multiple disease phenotypes correlate with ANRIL expression. PLoS Genet. 2010;6:e1000899.

9. Gil J, Peters G. Regulation of the INK4b-ARF-INK4a tumour suppressor locus: all for one or one for all. Nat Rev Mol Cell Biol. 2006;7:667-77.

10. Yap KL, Li S, Munoz-Cabello AM, et al. Molecular interplay of the noncoding RNA ANRIL and methylated histone $\mathrm{H} 3$ lysine 27 by polycomb CBX7 in transcriptional silencing of INK4a. Mol Cell. 2010;38:662-74.

11. Bochenek G, Hasler R, El Mokhtari NE, et al. The large noncoding RNA ANRIL, which is associated with atherosclerosis, periodontitis and several forms of cancer, regulates ADIPOR1, VAMP3 and C11ORF10. Hum Mol Genet. 2013;22:4516-27. 
12. Folkersen L, Kyriakou T, Goel A, et al. Relationship between CAD risk genotype in the chromosome 9p21 locus and gene expression. Identification of eight new ANRIL splice variants. PLoS ONE. 2009;4:e7677.

13. Bochenek G, Hasler R, El Mokhtari NE, et al. The large noncoding RNA ANRIL, which is associated with atherosclerosis, periodontitis and several forms of cancer, regulates ADIPOR1, VAMP3 and C11ORF10. Hum Mol Genet. 2013;22:4516-27.

14. Leppek K, Schott J, Reitter S, Poetz F, Hammond MC, Stoecklin G. Roquin promotes constitutive mRNA decay via a conserved class of stem-loop recognition motifs. Cell. 2013;153:869-81.

15. Negishi Y, Nishita Y, Saegusa Y, et al. Identification and cDNA cloning of single-stranded DNA binding proteins that interact with the region upstream of the human c-myc gene. Oncogene. 1994;9:1133-43.

16. Kotake $\mathrm{Y}$, Nakagawa $\mathrm{T}$, Kitagawa $\mathrm{K}$, et al. Long non-coding RNA ANRIL is required for the PRC2 recruitment to and silencing ofp15(INK4B) tumor suppressor gene. Oncogene. 2011;30:1956-62.

17. Holdt LM, Hoffmann S, Sass K, et al. Alu elements in ANRIL non-coding RNA at chromosome 9p21 modulate atherogenic cell functions through trans-regulation of gene networks. PLoS Genet. 2013;9:e1003588.

18. Koh CM, Sabo A, Guccione E. Targeting MYC in cancer therapy: RNA processing offers new opportunities. Bioessays. 2016;38: 266-75.

19. Ho JS, Ma W, Mao DY, Benchimol S. p53-Dependent transcriptional repression of c-myc is required for G1 cell cycle arrest. Mol Cell Biol. 2005;25:7423-31.
20. Qi L, Cornelis MC, Kraft P, et al. Genetic variants at $2 \mathrm{q} 24$ are associated with susceptibility to type 2 diabetes. Hum Mol Genet. 2010;19:2706-15.

21. Fehrmann RS, Jansen RC, Veldink JH, et al. Trans-eQTLs reveal that independent genetic variants associated with a complex phenotype converge on intermediate genes, with a major role for the HLA. PLoS Genet. 2011;7:e1002197.

22. Zeller T, Wild P, Szymczak S, et al. Genetics and beyond--the transcriptome of human monocytes and disease susceptibility. PLoS ONE. 2010;5:e10693.

23. Heinzen EL, Ge D, Cronin KD, et al. Tissue-specific genetic control of splicing: implications for the study of complex traits. PLoS Biol. 2008;6:e1.

24. Zou F, Chai HS, Younkin CS, et al. Brain expression genomewide association study (eGWAS) identifies human diseaseassociated variants. PLoS Genet. 2012;8:e1002707.

25. Takai T, Nishita Y, Iguchi-Ariga SM, Ariga H. Molecular cloning of MSSP-2, a c-myc gene single-strand binding protein: characterization of binding specificity and DNA replication activity. Nucleic Acids Res. 1994;22:5576-81.

26. Yue W, Sun LY, Li CH, Zhang LX, Pei XT. Screening and identification of ovarian carcinomas related genes. Ai Zheng. 2004;23:141-5.

27. Chisa JL, Burke DT. Mammalian mRNA splice-isoform selection is tightly controlled. Genetics. 2007;175:1079-87.

28. Zhang EB, Kong R, Yin DD, et al. Long noncoding RNA ANRIL indicates a poor prognosis of gastric cancer and promotes tumor growth by epigenetically silencing of miR-99a/miR-449a. Oncotarget. 2014;5:2276-92. 